\title{
L'induction : une notion qui brouille les circuits et envoie du bruit sur la ligne
}

\section{Françoise Colin}

\section{(2) OpenEdition}

12 Journals

Édition électronique

URL : http://journals.openedition.org/communicationorganisation/2009

DOI : 10.4000/communicationorganisation.2009

ISSN : $1775-3546$

Éditeur

Presses universitaires de Bordeaux

\section{Édition imprimée}

Date de publication : 1 novembre 1997

ISSN : 1168-5549

\section{Référence électronique}

Françoise Colin, «L'induction : une notion qui brouille les circuits et envoie du bruit sur la ligne », Communication et organisation [En ligne], 12 | 1997, mis en ligne le 19 décembre 2012, consulté le 20 avril 2019. URL : http://journals.openedition.org/communicationorganisation/2009; DOI : 10.4000/ communicationorganisation.2009

Ce document a été généré automatiquement le 20 avril 2019.

(c) Presses universitaires de Bordeaux 


\title{
L'induction : une notion qui brouille les circuits et envoie du bruit sur la ligne
}

\author{
Françoise Colin
}

1 Enseignant la communication dans plusieurs cursus universitaires, je suis toujours étonnée de l'insistance avec laquelle les étudiants répètent le schéma de Shannon comme un point de départ à la compréhension de toute situation de communication. En y regardant de plus près, la chose est fort compréhensible: la plupart des manuels pédagogiques destinés aux étudiants de ces filières présentent ce shéma dès leurs premières pages pour le tempérer ensuite par des apports chronologiquement plus récents et épistémologiquement fort différents (un peu de Palo-Alto pour introduire le non-verbal par exemple), mais la superposition d'énoncés de théories différentes n'efface pas la première "scène » introduite, et invariablement c'est à partir des termes « émetteur » « récepteur » « canal » qu'ils attendent l'analyse des situations rencontrées au cours des exercices...

2 Aussi la proposition de l'équipe du Grec/o de prolonger sa recherche par un colloque interrogeant la notion d »'Induction » à l'œuvre dans la communication, a-t-elle été pour moi l'occasion de faire le point sur ce qui dans nos schémas mentaux s'oppose à une pensée inductive et résiste en utilisant dès que possible les modèles linéaires et déductifs.

3 À l'école on nous avait appris que l'induction était un mode de raisonnement dangereux car comportant un risque de subjectivité, un manque de rigueur qui serait fatal à notre présentation, la classant définitivement dans le «non-scientifique », l'approximatif et l'amalgame obscur entre éléments non fondés...

4 La Déduction seule avait droit de cité. La déduction était comme un entonnoir : à partir d'hypothèses crédibles ou de phénomènes précis analysés séparément, on pouvait déduire une loi qui expliquait avec la rigueur nécessaire la nature des effets observés et promettait la répétition de ces mêmes effets dès que les mêmes causes seraient à nouveau réunies. 


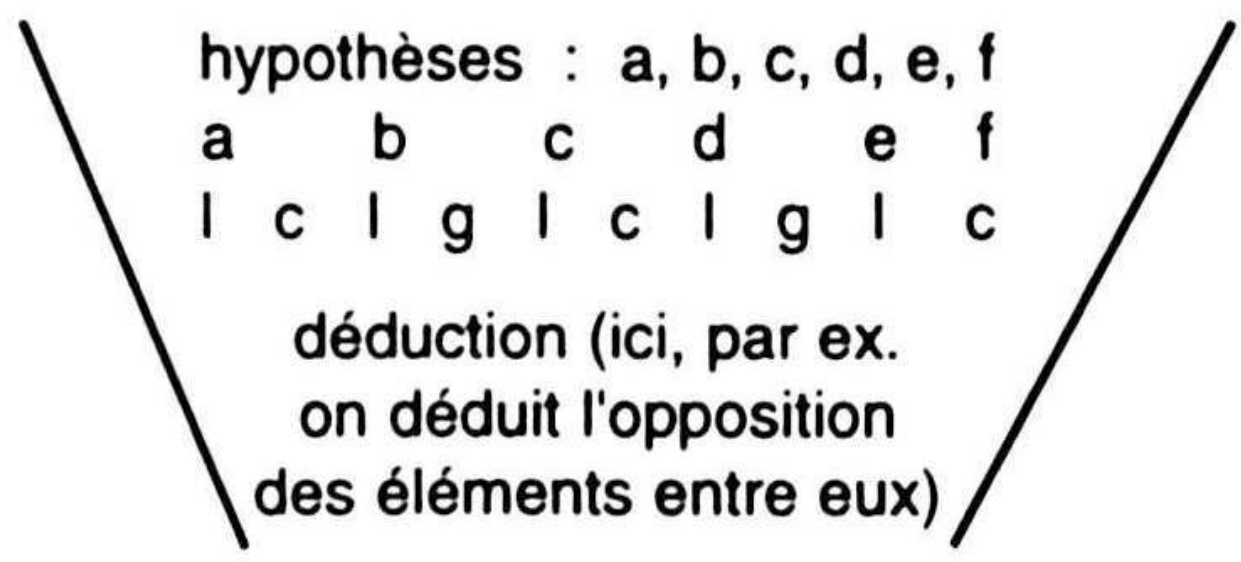

\section{Raisonnement hypothético-deductif}

Par contre, l'induction était présentée comme un raccourci dangereux parce qu'issu de l'intuition, sa soeur jumelle, et partageant les mêmes anathèmes. Elles proposaient de partir d'un cas particulier constaté pour supposer qu'il se reproduirait de même lorsque les mêmes circonstances seraient réunies. De là à en tirer une loi, il n'y avait qu'un pas qu'il fallait se garder de franchir à moins de soumettre les faits en question à l'épreuve de la déduction.

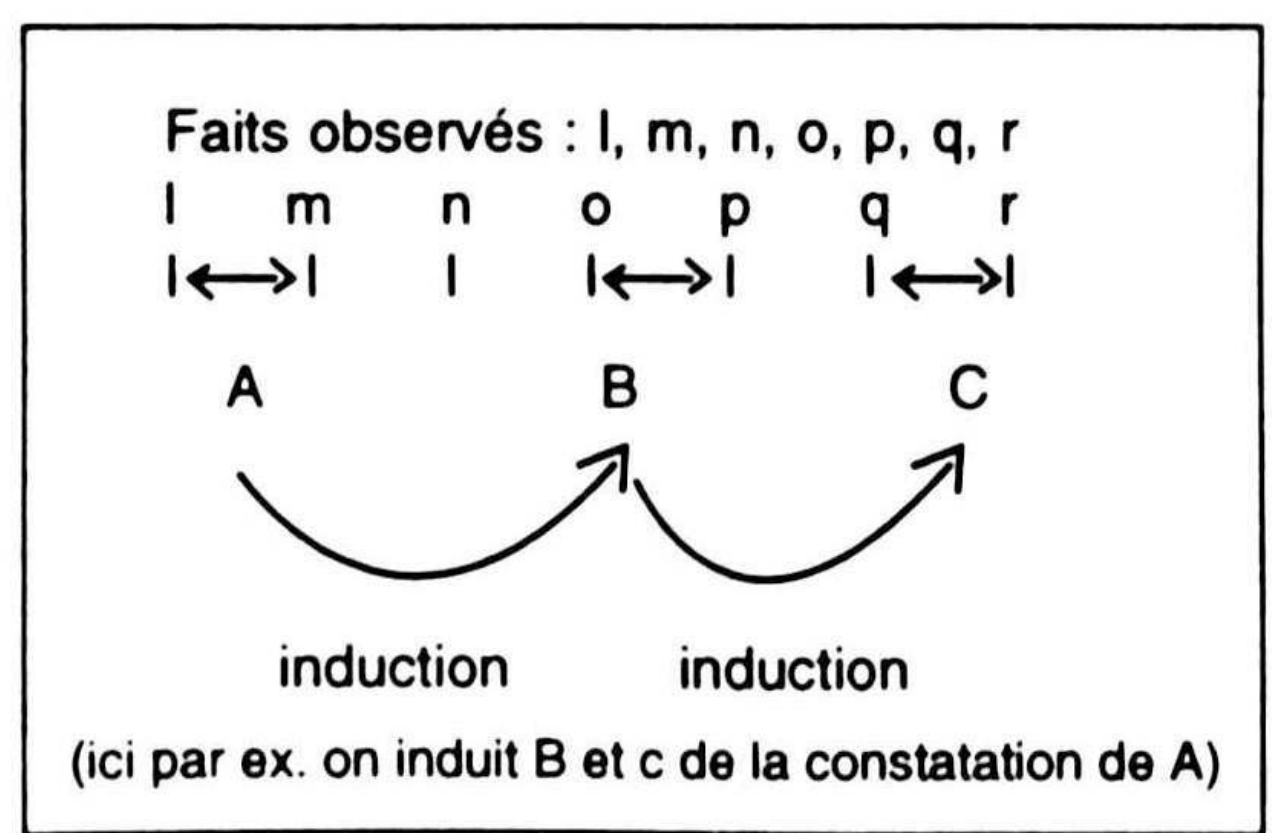

\section{Raisonnement par induction}

Si les sciences « dures » qui produisent du vérifiable, du répétable, mais qui produisent en même temps le modèle du seul mode de cheminement mental acceptable par l'ensemble d'une communauté, ont imposé la déduction comme type de raisonnement dominant, il est alors compréhensible que tout domaine de connaissance ait tenté de s'y conformer. Compréhensible, mais pas forcément légitime. Car la science est un « médium » : un mode 
de représentation par lequel le sujet appréhende le monde, et il n'y a pas qu'un seul chemin d'accès : les sciences autrefois dites " expérimentales ", les sciences humaines, les sciences de l'information et de la communication doivent être distinguées, et leurs modèles de pensée sont multiples et divers.

7 De plus, « la science en train de se faire » fonctionne souvent à l'induction, à l'analogique mais le mode de communication : « la science en train de se dire » masque ce défaut sous l'inévitable séquence linéaire »théorie, observation, loi ».

8 Dans le domaine à présent dénommé «DMFO-COM », il semble qu'il y ait comme une nécessité d'administrer une preuve de scientificité, et que cette preuve doive passer par le recours à des modèles «mathématiques » ou linéaires comme celui de Shannon, ou du moins par des enchaînements de type déductif. Ces types de raisonnement ne fonctionnent-ils pas comme des rites d'accession au titre de science? Si c'est le cas, ce serait plutôt la marque d'un manque de maturité; ne vaudrait-il pas mieux trouver des modèles spécifiques féconds et différenciés s'appliquant aux divers champs du domaine dont la notion de « communication » est le trait unitaire ?

9 Nous allons évoquer rapidement les problèmes épistémologiques de la représentation scientifique en nous servant d'une opposition d'école entre le positivisme et le constructivisme qui ne recouvre peut-être pas exactement l'opposition entre les modes de raisonnement déductif et inductif ni non plus l'évolution chronologique de la pensée scientifique mais cela nous permettra:

10 - d'une part, de situer l'induction comme un mode de fonctionnement opératoire qui a toujours été présent, sinon dans la pensée scientifique, du moins dans la pratique des chercheurs, mais qui a souvent été occultée pour des raisons culturelles, - d'autre part de positionner la problématique du sujet.

11 Puis nous chercherons les raisons d'un entêtement, d'une résistance à l'acceptation de nouveaux processus de connaissance et de nouvelles formes de représentation non spécifiquement linéaires.

12 Enfin nous tenterons de montrer que l'échange entre deux sujets peut être analysée à partir d'autres modèles non-linéaires et de type inductif...

\section{La réalité et sa représentation: du positivisme au constructivisme}

13 Le problème est aussi vieux que la philosophie : il s'agit de situer le sujet par rapport au monde, et d'accorder un statut à l'indéfinissable « Réalité ». «La réalité qui fait face au sujet » diraient les positivistes, « la réalité dont nous faisons partie nous aussi au moment même où nous tentons de la saisir » diraient les constructivistes. En vérité, dans l'histoire de la pensée scientifique, la cassure n'est pas aussi nette. Ce qui est sûr c'est que le statut de la réalité est indissociable de la place que l'on accorde au sujet...

14 La représentation de la réalité comme un monde étendu devant le sujet qui, libre de sa pensée, se l'approprie en isolant les éléments et en les reliant ensuite par des règles formelles, inscrites, d'une certaine manière, dans les choses, est le postulat de la pensée cartésienne, fondatrice de la science moderne rationnelle, excluant le principe d'un ordre établi par la seule puissance divine. 
15 «Aux $\mathrm{XVII}^{\mathrm{e}}$ et $\mathrm{XVIII}^{\mathrm{e}}$ siècles la vérité des sciences de la Nature avait une force éminemment critique qui portait au grand jour la dimension sociale et politique de la pratique scientifique. (...) Il s'agissait d'opposer l'ordre de la Nature ou de la Raison à l’ordre de la Monarchie et de l'Église. N'accepter d'autres "vérités » qui viennent d'ailleurs, d'en haut, et qui sont imposées d'autorité. C'est aussi faire valoir la réalité contre les dogmatismes, les croyances, les préjugés et la magie des mots qui véhiculaient les valeurs en place et qui justifiaient l'ordre social $»^{1}$.

Il faut insister sur cet enracinement culturel du sujet face au monde et de la nécessité d'appliquer des règles déductives pour relier les choses entre elles: ces aspects extrêmement codifiés de l'esprit cartésien sont encore aujourd'hui, pour le chercheur qui travaille avec des scientifiques d'autres pays la marque, glorieuse croit-on, de l'« esprit français ». La nécessité de dominer la pensée sauvage, vécue comme dangereuse, appartient à nos mythes fondateurs de la science.

Mais il apparaît vite que cette réalité du monde devant le sujet est double : derrière les phénomènes apparents, il y a des phénomènes cachés (car nos sens peuvent être trompeurs) et seul le scientifique saura les retrouver grâce à ses constructions mentales. Le sujet et tout ce qui s'attache à la subjectivité est donc dangereux et risque de faire basculer la « vraie » science dans la fausse perception de la pensée magique.

Loupure entre subjectivité et objectivité qui était vécue comme nécessaire pour ne pas retomber dans l'obscurantisme a pu aboutir parfois à créer un monde théorique plus Réel que la réalité trompeuse qui nous entoure: de la réalité nous ne percevons finalement que l'ombre sur la paroi de la caverne où nous sommes enfermés et nous fonctionnons dans un espace Imaginaire où seul le monde des Idées est digne d'intérêt. Pour $\mathrm{Cl}$. Allegre ${ }^{2}$ c'est donc bien avant Descartes qu'il nous faut remonter, jusqu'à Platon, pour comprendre la fascination pour les idées abstraites, typiquement française, qui a conduit nombre de scientifiques à ignorer superbement la place pourtant prise dans leurs propres recherches par l'expérimentation et l'observation.

Cette coupure entre subjectivité et objectivité a obligé les sciences à ignorer ce qui leur était extérieur, à se clore sur elles-mêmes : " l'idéal du XVII ${ }^{\mathrm{e}}$ et du XVIII ${ }^{\mathrm{e}}$ flotte toujours au-dessus de la pratique scientifique, mais il n'est plus lié à cette pratique, il n'est plus qu'un slogan académique et ornemental qui permet de faire oublier les conditions réelles où s'exercent les sciences aujourd'hui $»^{3}$

Le raisonnement déductif et la suprématie de la théorie sur l'observation ont donc été les arcanes de la pensée scientifique qu'A. Comte a officialisée il y a plus d'un siècle. La classification d'Auguste Comte plaçant les mathématiques, royaume de l'abstraction, en haut de l'échelle conforte bien ce primat du monde Idéel platonicien; la position des sciences expérimentales au deuxième rang n'a pas peu contribué à ce rejet de la valeur de l'observation comme de l'induction qui président toutes deux à la créativité de la recherche. Déplorant cette place prise par les mathématiques dans l'ensemble du système éducatif, Allegre y voit là un signe de la non-fécondité actuelle de la recherche en France. Les élites dirigeantes ont été - en France - sélectionnées par les mathématiques «la prééminence des mathématiques, c'est le refus du réel au cœur même du système éducatif donc de tout notre système de sélection »...) « Nous ne savons pas former nos jeunes au véritable esprit scientifique: observation, rapprochement de deux faits que tout éloigne, imagination, créativité... ${ }^{4}$ 
21 Les sciences dites naturelles ou expérimentales ont dû construire un néopositivisme; moins abstraites, elles ont à leur tour mis en œuvre des procédures linéaires de déduction, plaçant l'observation sous la coupe idéologiquement inattaquable d'une théorie à vérifier : théorie $\rightarrow$ observations $\rightarrow$ loi. Mais combien de découvertes faites par hasard (un hasard favorisé, bien sûr, par un regard "préparé » à le saisir disait Pasteur), par rapprochement intuitif et par analogie seront ensuite "communiquées" officiellement selon cette démarche hypothético-déductive, longtemps la seule forme officiellement admise dans les assemblées savantes?

Bachelard n'a fait que renforcer la place du sujet dans le dispositif en montrant que si les scientifiques se contentaient d'observer la terre et les étoiles, ils ne verraient strictement rien à moins de posséder d'avance sur leurs papiers des théories, des trajectoires, des calculs : c'est avec les « yeux » de la science qu'ils « voient » que l'étoile du berger et celle du matin n'en font qu'une et qu'il s'agit de Vénus, une planète...

Ce concept a, dans les années soixante, fonctionné comme une nouvelle norme du comportement scientifique. Lorsque les sciences humaines se battaient pour délimiter leur champ et mériter le nom de science, le "saut épistémologique » ou mieux la " coupure épistémologique » avait valeur de castration permettant d'accéder à cette deuxième réalité garantissant la scientificité d'une recherche sociologique ou ethnologique. La preuve que le saut avait été accompli, que le rubicon était franchi n'était souvent que le discours lui-même, ou plutôt une certaine façon de le mettre en forme. L'évidence non-contestable d'un regard épistémologiquement garanti s'est imposée là, appauvrie, dans un cadre où l'accession au titre de science posait problème et où les discours tentaient de se conformer à l'académisme en vigueur. Les objets d'études des sciences humaines: l'homme, les rapports sociaux, la culture, etc., avaient permis de montrer l'importance de la subjectivité dans le regard scientifique et le positionnement du savant comme observateur « humain ». Ce qui rendait plus évident un décalage qui a, néanmoins, toujours existé entre la "science en train de se faire » et la «science en train de se dire ", de « se mettre en scène ».

24 Les sociologues des sciences qui, analysent aujourd'hui les pratiques scientifiques et les processus de découverte comme des objets d'études « ethnologiques » montrent l'écart qui existe entre les échanges verbaux dans une équipe au moment de la découverte et les discours « officiels » codifiant cette même découverte lors d'assemblés savantes-

Bruno Latour, dans un article intitulé «Introduction à l'anthropologie des sciences et des techniques $\|^{5}$ resitue les pratiques des chercheurs : «le savant ne regarde pas les étoiles » semble-t-il répondre à Bachelard, « mais l'image en couleur artificielle que l'ordinateur a recomposé à partir de l'image optique ». Il fixe des formes, il recompose les traces, il fait varier les échelles, il mathématise, bref il travaille sur le «médium-science » lui-même jusqu'à ce que « l'exactitude glisse du médium vers le message »...

26 «Bruno Latour et Serge Woolgar analysent la science en train de se faire comme une activité rhétorique. Le chercheur doit avant tout persuader.(...) Un nouvel énoncé ne deviendra un fait scientifique que si le chercheur réussit à convaincre ses collègues, à éviter que ceux-ci lui rétorquent «mais tu pourrais aussi bien dire que... » et proposent des énoncés également probables. (...) Pour imposer un fait, le chercheur utilisera toutes les ressources de la rhétorique (...). Les éclairs de génie s'expliquent le plus souvent par le hasard des circonstances, les aléas des rencontres, des discussions avec les collègues. L'explication par l'idée nouvelle est le plus souvent reconstruite après coup, par le savant 
et son historiographe. Mais quand par hasard, l'ethnographe est là pour observer la science en train de se faire, il peut observer tout ce que les éclairs de génie doivent aux circonstances. Au bout d'un certain temps l'énoncé scientifique non contesté est réifié, il s'intègre aux savoirs scientifiques du moment, il devient une boite noire, un fait scientifique stable considéré par chacun comme faisant partie de la nature. $»^{6}$

Dans le même ordre d'idées, Vinciane Despret nous apporte l'humour d'un regard ethnologique sur une petite communauté d'ornithologues en train d'observer un extraordinaire oiseau du désert israëlien «le cratérope écaillé ». Les savants observent analysent et attribuent oralement, au hasard de leur propre vision très anthropomorphique des causes aux comportements de «danse » de ces étranges oiseaux pour finalement rédiger un article très conforme scientifiquement à ce que les revues américaines autorisent, en l'occurence la revue de l'Université de Cambridge. ${ }^{7}$

Les actes d'un séminaire intitulé "Technologie et Société » et rassemblés sous le titre "Question de méthode » par la revue Réseaux en 1988 (CNRS-CNET) font état d'analyses des "pratiques artisanales» des chercheurs. Louis Quéré, entre autres, y traduit une recherche maintenant célèbre de Garfinkel ${ }^{8}$ dans laquelle il analysait, en 1967, le moment de la découverte du pulsar optique par quatre physiciens. Quéré refuse de traduire le terme d'accountability, expliquant qu'il s'agit du concept clé de Garfinkel. Garfinkel s'est attaché à montrer que :

- « l'objet découvert (le pulsar optique) est façonné par le travail in situ et en temps réel des astrophysiciens au cours de leur nuit de travail,

31 - que l'objet découvert consiste strictement en ce travail comme l'objet du potier qui prend forme entre ses mains et dépend de la manière de l'élaborer.

- comment cet objet en vient à exister comme objet naturel indépendant de l'activité pratique qui l'a construit, son statut d'objet culturel étant occulté, cette occultation étant socialement organisée,

33 - comment par la suite les chercheurs rendront compte de leur recherche en travestissant leurs pratiques de fait et en ne décrivant leur travail qu'en mettant en scène l'objet produit comme s'il était objectivable-en-soi et analysable-en-soi. Les schèmes culturels d'interprétation et de narration sont des théories réifiantes produisant de nouveaux « accounts » (p. 110).

34 L'accountability est perçue comme une courbure de l'espace qui infléchit l'environnement (équipement technique, savoir-faire, schèmes de compréhension) du chercheur et lui permet l'intelligibilité de son objet de recherche. L'accountability ${ }^{9}$ est réflexive, c'est-à-dire que les éléments du cadre de recherche informent les activités du chercheur et ces dernières informent à leur tour « l'environnement » et cela permet une transformation permanente. Il ne s'agit pas d'un déterminisme mais d'une interaction. L'environnement fait agir, donne à penser, induit des effets...

35 Le fait de traiter une chose comme un objet défini implique donc une abstraction considérable qui oblige le chercheur à sélectionner quelques-uns des multiples éléments de son champ d'expérience. Et pour le transmettre comme "connaissance", il doit mobiliser son savoir des structures sociales, des discours considérés comme légitimes... L'environnement fait agir, donne à penser, induits des effets... Bref, le chercheur doit « construire » en permanence selon un processus qui, pour nous n'est rien d'autre que l'induction à l'œuvre dans la science elle-même. 

sur la notion de modèle en biologie, et surtout J. Piaget, provoquant à partir de sa réflexion sur la psychologie cognitive, le développement d'une construction épistémologique qu'il appellera d'abord "génétique ", suscitèrent une reformulation d'une épistémologie concurrente des positivismes, que J. Piaget proposa d'appeler le constructivisme : à l'axiomatique positiviste fondée sur la réalité ontologique de l'objet analysable indépendamment du sujet qui l'observe, J. Piaget proposa de substituer une axiomatique constructiviste fondant la science sur des projets de connaissance plutôt que sur des objets : la connaissance est processus avant d'être résultat, et elle ne s'entend que dans les interactions du sujet et de l'objet ». (...). «Modification radicale qui pouvait rendre possible l'appel à une Logique Non-Aristotélicienne telle que l'avait formulée Korsybski en $1930 »^{10}$. Selon Le Moigne, H. A. Simon, H. Von Foerster, G. Bateson, E. Morin, P. Watzalwick contribueront notamment à l'édification de cette epistemologie constructiviste. s'appuierait pas sur la pensée linéaire ni les déterminismes trop strictement entendus comme des rapports causes $\rightarrow$ effets et qui admettrait le réalisme d'un monde objectif : «les propriétés du monde ne sont pas des faits de conscience ou d'imagination: elles appartiennent bien au monde objectif. Pour ce "réalisme objectif», la notion de réalité en soi n'a pas grand sens : une chose émerge comme un objet défini, significatif ou pertinent, quand elle est déterminée dans le cours d'une interaction avec des agents doués d'une capacité de perception, de sélection de manipulation et d'un pouvoir de symbolisation. $»^{12}$

41 L'idéologie dans la science n'est pas évacuée, elle est conscientisée, la société n'étant plus vécue sur le mode d'un déterminisme appliquant une force extérieure aux agents, mais faisant partie d'eux-mêmes. 
42 Garfinkel, (déjà cité) a créé le concept d'accountability pour «contourner » le déterminisme du contexte sur l'acteur et créer l'interactivité. P. Flichy ${ }^{13}$ développe dans son ouvrage une théorie de l'innovation technologique qui rejette le constructivisme trop linéaire de l'école française de sociologie des sciences et des techniques qui affirme que la technique est déterminée par le social (Latour). Ces chercheurs, ainsi que ceux que nous avons déjà cités (Le Moigne, Quéré) proposent une vision nouvelle du rapport entre subjectivité et objectivité : «Dès lors que la subjectivité est thématisée non plus comme une origine de l'action et de son sens mais comme aboutissement par appropriation (...) de l'action dans le cadre d'une interaction avec l'environnement, il n'y a plus de sens à rendre compte de l'action uniquement par la motivation des agents (..) ni par l'intériorisation des valeurs de la collectivité. Le paradigme de la communication rend alors possible une approche internaliste de la socialisation des conduites et des événements, en ce sens que l'intelligibilité de ce que les gens disent et font est socialement produite par une activité organisante de ces agents rapportant les actes et les paroles à un environnement social familier et supposé connu en commun avec les autres"

«Ce qui requiert des acteurs que dans leur activité organisante, ils mobilisent leur savoir de sens commun des structures sociales et honorent leurs attentes normatives réciproques d'arrière-plan, relatives aux opérations socialement organisées, qu'ils font les uns par rapport aux autres, aux définitions de l'ordre des choses qu'ils considèrent comme légitimes, aux cadres normatifs d'activités qu'ils sont supposés prendre comme base d'inférence et d'action $»{ }^{14}$

Cette citation appelle plusieurs remarques :

- lorsque les acteurs « mobilisent » leur savoir, lorsqu'ils « considèrent » leurs actions par rapport à « l'ordre des choses » quel est le processus cognitif qu'ils mettent en place pour « penser»? l'induction n'est pas citée mais elle semble évidente: comparer afin de reconnaître pour choisir et agir, repose sur des processus analogiques... Ceci n'est pas sans nous rappeler Garfinkel et l'accountability.

- la praxéologie ici définie n'annule pas l'objectivité scientifique, mais appelle une nouvelle définition du sujet.

47 Il n'y a plus de philosophie du sujet cartésien face au monde, et du même coup, la position du sujet en train de faire la science est devenu un peu «flottante » dans cette nouvelle épistémé : l'observation du chercheur présuppose sa transparence à lui-même. Mais sa participation à l'objet de la recherche le rend, un temps, opaque à lui-même; et l'oscillation entre ses deux mouvements nécessite de repenser le rôle du sujet scientifique. La différence entre la notion d'individu et celle de sujet doit être nettement posée; et il semble que la confusion persiste souvent. L'individu est un des objets des sciences sociales, et le chercheur peut s'observer comme individu à travers ses pratiques sociales et culturelles, ce qui ne dit rien sur sa position de sujet. Le sujet est un élément réflexif de la recherche, au même titre que l'environnement et les outils techniques de la recherche. De même, il est sujet lorsqu'il pense l'aboutissement des développements scientifiques qu'il met en place, sujet alors « moral ».

D'après Alain Renault, « il faudrait faire les frais d'une nouvelle philosophie du sujet: l'individu qui veut s'instaurer comme sujet ne peut le faire en «monade» leibnizienne mais en se pensant comme membre d'un monde d'humanité fait d'une infinité de sujets. (...) La notion de sujet comprend en elle l'intersubjectivité.$^{15}$ 
49 Avant de revenir sur cette notion de sujet, fermons notre tour d'horizon des processus du raisonnement scientifique en espérant avoir montré que le raisonnement déductif linéaire n'est qu'une des facettes du raisonnement scientifique, qu'il est historiquement datable, et que son utilité démonstrative n'a rien à voir avec la place idéologiquement démesurée qu'il occupe encore dans le panorama des «nouvelles sciences». Il fonctionne comme un rempart contre les peurs obscures d'un retour à la pensée magique et d'une incertitude quant à la position du sujet dans la science.

50 Le positivisme n'est pas une "erreur ", une étape historique "dépassée », c'est une position philosophique du sujet face au monde. Cette position de face à face est tellement ancrée dans notre culture que la corrélation entre le monde scientifique et le monde «quotidien » est restée grande jusqu'à Bachelard, et nous pensons que cette corrélation est une force d'inertie qui a bloqué et bloque encore l'évolution de la pensée scientifique à ce stade. Il y a donc eu instauration d'une idéologie puissante contraignant le discours scientifique à passer sous ces fourches caudines.

51 Le positivisme a fini par se fissurer devant des phénomènes difficilement représentables indépendamment de l'observateur et ces phénomènes n'étaient pas ceux des sciences humaines au départ, il s'agissait la physique quantique, de l'astronomie, de la microbiologie, bref de l'infiniment grand et de l'infiniment petit qui n'obéissent pas aux mêmes règles : la connaissance du minéral n'explique pas la géologie et celle de la goutte d'eau n'explique pas la houle. La notion de temps et d'espace est elle-même bouleversée « Il faut s'y faire, nous vivons dans un monde non linéaire, la droite ne gouverne pas la nature, les courbes fractales y sont la règle. La complexité des comportements peut résulter de peu de facteurs mais non linéairement combinés ${ }^{16}$. Les outils eux-mêmes appelaient de nouveaux raisonnements.

52 On a pu voir qu'il y avait déjà "construction » dans le positivisme : lorsque A. Comte déclare «le positif, c'est le réel $»^{17}$ cela peut paraître paradoxal si l'on songe au dédain dans lequel était tenu tout raisonnement qui observerait la réalité sans avoir formé de théorie préalable à l'observation. Mais ce serait ignorer la différence entre la "réalité " trompeuse, soumise à notre imagination, et le « réel » constitué par l'ensemble des lois qui lient les choses entre elles et que le scientifique s'emploie à retrouver.

53 Ce réel-monde était donc théorique et construit et la marque du positivisme reste le raisonnement hypothético-déductif linéaire qui autorise tous les déterminismes et aussi son intransigeance et l'instauration de ses modes de raisonnement en idéologie. Quant à la position du sujet, elle reste la différence fondamentale entre les deux approches.

Il n'y a pas d'un côté la connaissance et de l'autre un ensemble de procédures pour la manipuler: La science, comme le langage, est une médiation conçue, selon les époques culturelles soit comme un moyen entre le sujet et le monde, soit comme une synergie mettant en continuum le sujet et le monde. L'alternance entre l'observation, l'expérience et la théorie ne jette pas la rigueur scientifique aux orties, mais la science n'est déductive que sur un court chemin et l'induction fait partie intégrante de cet aller retour fécond entre l'observation et la théorisation. La connaissance produit elle-même ses modes de pensée et ses outils qui à leur tour permettent cette connaissance. «Ce qu'une personne connaît est coextensif à l'ensemble des programmes dont elle dispose pour le manipuler $»^{18}$ 


\section{Le modèle de Shannon comme résistance de la linéarité}

Dans ces nouvelles perspectives «le modèle » est un moyen pour rendre compte des connaissances. Un modèle sera construit pour une recherche datable et finie et sera difficilement exportable. Le modèle apparaît comme une abstraction provisoire et pas toujours mathématisée qui a pour vocation de simplifier la complexité apparente et d'aller vers la conceptualisation.

Les sciences admettant différents univers juxtaposés rendent crédibles des modélisations différentes et adaptées à leurs recherches qui ne sont plus simplement des objets mais des processus, des relations. "J'ai parfois comparé les modèles à des navires. L'intérêt pour moi, le navire construit est de le mettre à l'eau, de voir s'il flotte, puis de lui faire monter ou descendre à mon gré les eaux du temps. Le naufrage est toujours le moment le plus significatif $» .^{19}$

Le modèle construit par Shannon en 1948 avait pour but l'adaptation du code au canal de transmission. Shannon travaillait sur le rendement des lignes téléphoniques au Laboratoire Bell. L'information était un élément nouveau du paysage scientifique, élément que l'on pouvait transformer en grandeur quantifiable afin de le coder et de le décoder.

Cette modélisation à partir d'observations techniques a permis l'opération de codage et de décodage, elle a été conçue dans le but d'obtenir l'annulation du bruit dans l'émission d'information. «Toute référence rigoureuse à la théorie de l'information implique donc que soit respectée la dimension mathématique originelle de son élaboration. Toute généralisation dans un champ hors de portée des mathématiques implique donc que soit reconnue explicitement le passage des notions qu'elle implique au statut de métaphores $\gg .^{20}$

9 Pourtant la réutilisation, à l'extérieur de ce champ, des notions comme «émetteur, récepteur" pour donner une vision de la communication en général et surtout le réemploi d'une sorte de causalité linéaire entre les notions est si fréquent qu'il y a là matière à s'inquiéter : qu'est-ce qui résiste à l'ouverture extraordinaire des situations de communications permettant une multiplicité d'autres modèles ? qu'est ce qui séduit dans la chaîne entraînante de ce modèle : émetteur $\rightarrow$ message $\rightarrow$ récepteur » qui fonctionne fortement ? Son fondement mathématique? mais il n'est jamais repris !

Est-ce la notion de système qui rend plus délicate la traduction des phénomènes? « Tout ce qui est simple est faux, tout ce qui est complexe est inanalysable » dit E. Morin.

61 L'analyse en termes de système et d'interrelations est souvent évoquée mais le déterminisme revient au galop. Ainsi alors que l'expérience quotidienne contredit sans arrêt cette chaîne, la présentation de la communication faite dans les manuels et les salles de cours passe et repasse par ce schéma qui est aussi incantatoirement répété au début de tout manuel à l'usage des étudiants en communication, même lorsqu'il s'agit de traiter de la Psychologie de la communication ${ }^{21}$, ce qui ne facilite pas l'ouverture vers un autre mode de compréhension des phénomènes de la communication.

Cela évoque ces images en trois dimensions qui sont proposées dans les magazines pour adolescents : à première vue ce ne sont que des tâches de couleurs avec une certaine régularité, mais à y regarder mieux - ou plutôt en les regardant autrement - on voit 
soudain une image en relief se détacher, sphinx ou personnage. Pour y arriver, il faut "désaccomoder " lentement sa vision et focaliser sur deux points jusqu'à ce qu'ils se confondent, ce que nos yeux font naturellement lorsqu'on est fatigué... Quand on distingue l'image cachée, elle est très nette, en relief, se détachant sur un fond de couleur, mais si notre regard la quitte, tout se brouille, et il faudra faire à nouveau l'effort de refocalisation pour la retrouver. De même, sur ces notions, on "défocalise " très vite comme si la linéarité s'imposait «naturellement", comme si la compréhension n'était que par éclairs...

63 Il faut dire que la présentation de ce modèle a été faite par Shannon sous une forme qui la met totalement en congruence avec la pensée linéaire et déterministe :

64 - il isole des unités simples et rassurantes : «émetteur-récepteur-message » dans ce qui est vécu quotidiennement comme confus : la communication.

65 - on le sait, il est par ailleurs mathématisable donc crédible, et cela sauvera, peut-être, les sciences de l'info-com des balbutiements de l'enfance.

66 - dans la linéarité cause -> effet nous retrouvons, rassurés, la flèche du temps qui est un paradigme constitutif de nos représentations occidentales. Notre façon corporrelle, quotidienne de vivre ce cadre du temps n'aide pas à le transgresser. Les civilisations qui ont du temps une représentation circulaire (Amérique centrale, Inde...) ont-elles la même rhétorique de raisonnement?

67 - sur cette chaîne qui ressemble à une chaîne causale, il est facile de "glisser " vers un déterminisme : l'émetteur est la cause du message, le récepteur n'en capte que les effets etc....

68 Nous avons vu dans notre première partie la force avec laquelle ces éléments ont étés imposés dans l'ensemble du système scolaire et scientifique pendant des générations. Il n'est pas étonnant que la résistance soit grande à essayer d'adopter des modes de compréhension qui soient plus près des expériences de communication que nous vivons tous les jours et qui n'aient rien à faire avec la syntaxe des ingénieurs qui travaillent sur le bruit et non sur le sens...

69 Entre l'ordre symbolique nécessaire à Shannon, et celui imaginaire et fluctuant que nous venons de décrire comme présidant à la communication humaine, il ne peut y avoir la guerre : ce ne sont pas les mêmes finalité qui sont recherchées.

70 Dans les TIC le message n'a rien à faire avec ce que nous appelons un message dans la vie courante: c'est une suite de signes dont il faut mettre au point très précisément la syntaxe. L'informatique est une technique de la syntaxe: elle peut nous donner des procédures; les cybernéticiens, cogniticiens et autres chercheurs en intelligence artificielle ont besoin de règles d'inférences que l'on pourra transmettre à la machine. Le problème de la sémantique donc du sens du discours n'est pas un problème qu'ils se posent tous les jours, et quand ils le font, ils cherchent alors effectivement à représenter des faits sous forme de symboles. La vision dualiste du monde est un bon outil pour ce genre d'opération. Ils créent ainsi un système symbolique qui approchera le sens mais qui ne sera pas du sens car le sens n'existe pas en soi : il n'existe que pour qui le veut, le lit, le prend, le rejette, il n'existe que dans l'acte de communication que la machine ne peut pas faire, qui nécessite un sujet. 


\section{Modèles inductifs}

71 Face à la multiplicité de fonctions de la communication il est évident que d'autres schémas peuvent être proposés.

72 Prenons comme exemple une simple émission d'information sans dialogue : notons qu'il s'agit déjà d'interaction. Cette situation admet plusieurs formulations, en voici une :

73 A, que nous éviterons d'appeler Émetteur veut envoyer une information de nature « $\mathrm{i}$ » à $B$ (Récepteur ?). La nature même de cette information se transforme dès que A parle ou écrit ou tape sur son clavier et qu'il pense à $B$, car selon l'image qu'il a de B et l'image qu'il pense que $B$ a de lui (qu'ils se connaissent ou pas) et son désir de la conforter ou de l'annuler, le message va se modifier en s'émettant. Le feed-back c'est déjà cela : ce n'est pas le retour d'une information lancée, c'est l'envoi depuis un B, qui n'a dit mot, « d'émanations » même pas non-verbales mais tenant au fait même de l'existence de B qui vont transformer l'intentionnalité du message de A et, par petites touches, le guider vers un message « $\mathrm{i}$ » ou i'ou $\mathrm{j}$ tout différent de celui qu'il aurait envoyé à $\mathrm{X}$ ou Y. Si $P$ et seulement A a parlé peut-on dire que B n'a été que Récepteur? D est en tout cas responsable d'une transformation du message qui s'est opérée en même temps qu'il le « recevait ». Le récepteur a déjà induit par sa présence une transformation.

Qui n'a jamais bafouillé en laissant un message sur un répondeur téléphonique ? l'absence du récepteur attendu étant remplacé par un autre récepteur nous oblige à transformer illico la teneur du message. Qui dira que ce récepteur artificiel n'a pas agit comme un feed-back? Notre message se « déshumanise », perd tout contenu phatique pour devenir strictement informatif, comme dans tous les premiers échanges homme-machine.

«L'homme commence à hésiter sur les critères de sa propre humanité dès l'instant qu'il «communique » avec un Autre où il est en passe de reconnaître une pensée sans sujet. Que cet objet devienne le modèle de son propre fonctionnement cognitif (Mc Culloch) et une boucle étrange va se refermer : si l'automate n'est pas un homme, l'homme ne seraitil pas un automate ?22 Cet exemple nous démontre a contrario que, lorsque l'autre est humain ou humanisé (on peut fort bien humaniser la machine au bout d'une certain temps, mais elle ne sera jamais un " autre » car elle ne postule rien sur nous), l'échange est fait d'une représentation de soi et de l'autre qui se crée en même temps que la production du sens (phénomène que l'on pourrait tenter d'isoler comme « spécifique » de la communication) mais qui en est indissociable, comme le sujet ne peut pas se soustraire à la présence de l'autre qui est en lui et qui modifie en permanence l'acte de communication.

76 Ce phénomène d'ajustement qui préside à l'échange est permanent, il est de l'ordre de l'induction. C'est expérience analogique de la relation à l'autre qui détermine le sens du discours et qui leste tout échange pour en faire un langage humain. L'induction va déterminer le sens du message, le moment de l'émission et va « brouiller » la ligne...

77 Le seul schéma possible de ces rapports - si véritablement il est nécessaire de faire une représentation (donc de créer un modèle) - serait celui qui relierait A et B par une bande tournante qui n'aurait pas de fin - ce qui est déjà le cas du cercle, mais qui subirait des torsions dès que l'intentionnalité de d'envoyer un message à quelqu'un se fait jour: l'anneau de Moebius pourrait en être une représentation facile à tracer sur un tableau noir à condition de ne pas le comprendre comme le «canal» du message mais comme le 
symbole de la déformation inhérente à l'acte de communication le symbole du mouvement permanent à l'œuvre dans l'échange humain : transformation du message, décentrement du sujet, de sa représentation de lui et de l'autre... Il n'y a pas doublement de la représentation des choses dans un autre ordre qui est celui du langage : il y a un autre réel en train de se faire : l'acte de communication.

Dans cet acte A et B sont à la fois, on l'a compris, objet et sujet, non pas alternativement mais en même temps.

Allons plus avant : l'adaptation permanente nécessaire à laquelle se livre le sujet dans cet échange ne provoque-t-elle pas un dédoublement, comme un vacillement permanent de son engagement dans l'acte? A la suite de Pierce, Eliséo Véron nous propose un autre modèle tenant compte de ce dédoublement du sujet qui ajuste son action: "Toute opération de production de sens entre un acteur A et un acteur B présuppose la construction d'un tiers: C ». Si cette situation est sous le regard d'un observateur cherchant à l'analyser alors Eliséo Véron lui donne un autre nom : 0 , mais il nous laisse entendre que cette situation d'observation est toujours déjà là, il y aurait donc une équivalence entre $\mathrm{C}$ et $\mathrm{O}$. «L'indécidabilité peut en fait devenir visible, pour $\mathrm{A}$ ou pour $\mathrm{B}$, à tout moment: il suffit qu'il se mette à la place $O$ (observateur), c'est-à-dire qu'il se mette hors jeu, autrement dit encore, qu'il mette en suspens sa croyance $»^{23}$.

Le principe de la production du sens dans le discours est complètement différent de celui de la reconnaissance de ce même sens, et ce décalage perpétuel est à l'œuvre à tous moments. Pour lui, c'est ce décalage dans la circulation signifiante qu'il nous faut analyser si nous voulons comprendre comment évoluent les représentations sociales par la communication (entendue comme production de sens :

81 Cette analyse d'Eliséo Véron qui prend totalement en compte les phénomènes inductifs semble difficilement applicable dans des recherches concrètes comme celles qui nous sont soumises et qui portent le plus souvent sur la recherche de l'efficacité des communications dans les entreprises et les organisations. Pourtant il y a dans cette optique des études qui se mènent...

Sergio Vasquez Bronfman ${ }^{24}$ a été amené à analyser les échanges entre les acteurs d'une entreprise; il s'appuie sur la taxinomie proposée par John Searle pour l'analyse conversationnelle permettant de prendre en compte l'induction dans l'intentionnalité. Sur cette base, il construit un ensemble de boucles de circulation des informations. Au lieu de considérer le réseaux de communications comme un réseaux de nœuds (c'est-àdire d'émetteurs-récepteurs) reliés par des liens, il considère un réseaux de liens (teneur du message en fonction de l'intentionnalité) reliés par des nœuds. La linéarité cède le pas devant une analyse complexe dont la richesse vient de ce que chaque lien circule avec son intentionnalité et ses dérives qui permet de détecter l'inadéquation des liens au lieu d'attribuer au seul émetteur la cause de l'inefficacité de la circulation de l'information.

Ce modèle, cité juste pour mémoire, dépasse le cadre de notre analyse d'aujourd'hui car c'est un modèle social et nous quittons la référence épistémologique qui était le nôtre tout au long de cette interrogation. La notion d'individu pris dans un "arrière plan " (Searle) social avec toutes les épaisseurs d'images de soi de l'autre, du non-verbal du contexte déterminant, etc., n'était pas de notre propos, bien que nous puissions y retrouver l'induction comme déterminant les comportements; nous préférons en rester ici au plan philosophique du sujet et des systèmes symboliques qu'il a pu constituer. 
84 Nous avons ici voulu apporter un éclairage sur d'autres modèles possibles du schéma communicationnel de production de sens, car nous « intuitionnions » que le sujet dans ce schéma se guidait par des ajustements reposant sur des raisonnements plutôt de type analogiques et inductifs; mais un obstacle de taille s'est dressé : pourquoi une telle hésitation à reconnaître ces modes de raisonnements? Pourquoi les chercheurs continuent - ils à utiliser un "mode de comprendre $»^{25}$ qui exclut par là même un ordre de phénomènes pourtant perceptible par tous, et qui d'être exclu, rend caduques leurs analyses. «Le savoir lié par une cohérence formelle ne couvre pas tout le champ de l'expérience humaine (...). Il y a du vrai qui n'est pas saisissable dans un savoir lié $»^{26}$. Il a donc fallu analyser rapidement ce qui, dans nos socles de pensée, relève d'une é pistémologie positiviste afin de comprendre les résistances à l'application de ces notions et le refoulement culturel de la notion d'induction.

La pensée scientifique, présentée au début de notre propos, comme un système de représentation de la réalité, se retrouve, dans l'analyse systémique, comme un élément de plus dans le projet de compréhension du sujet, un élément en relation (c'est-à-dire en modification permanente) avec les autres éléments: environnement, outils, culture, intentionnalité... Les systèmes symboliques ou modèles qui peuvent être créés pour tel moment nécessaire à la compréhension, ne peuvent être déplacés sans être totalement modifiés par l'ensemble des autres éléments du champ nécessaire au nouvel acte de production du sens.

87 Car dans l'acte de communication on n'est plus dans un système symbolique ni représentatif : on est dans l'imaginaire c'est à dire dans un monde où les choses qu'il nous faut analyser sont des actes modifiables au fur et à mesure de leur création.

«La structure indécidable de l'imaginaire préside à toute production de sens $»^{27}$. Cette notion d'imaginaire est à comprendre comme réflexive et non comme un resurgissement de l'imaginaire platonicien car il n'y a pas d'un côté une perception qui serait de l'ordre de l'imaginaire, et de l'autre une construction théorique qui la dominerait. Il faut plutôt comprendre que le processus de production du sens est essentiellement pratique et s'ajuste en fonction des "images ", sans arrêt renvoyé d'un sujet à l'autre, sur les sujets eux-mêmes, sur la définition de la situation où ils se meuvent etc.

Et surtout la place du sujet dans une situation communicationelle n'est pas fixe, elle est vacillante car il est d'une part, engagé dans l'acte et d'autre part, en train de rassembler les éléments du champ (de « l'arrière plan » dirait Searle) pour guider la poursuite de son acte. Tant que l'on considérera le sujet comme un point fixe, un nœud sur un réseau, un aboutissement ou un départ, on restera dans une analyse déterministe, et on exclura la possibilité de comprendre la complexité d'une situation de communication.

\section{NOTES}

1. H Rose, S Rose et alii. L'idéologie de/dans ta Science. Seuil. 1977.

2. Claude Allegre. La défaite de Platon. Seuil. 1995 
3. H. Rose et alii, L'idéologie de/dans la science. Seuil, 1977, p. 263.

4. Cl. Allegre idem note 2. p. 451.

5. Revue Culture technique, $\mathrm{n}^{\circ}$ 14, Juin 1985

6. Patrice Flichy. L'innovation technique, éditions La Découverte. Pariss 1995. Page 90 à 92.

7. Vinciane Despret. La danse du Cratérope écaillé ou ta naissance d'une théorie éthologique. coll. Les empêcheurs de penser en rond. Paris. 1996

8. Harry Garfinkel. "Travail d'une science découvrante». Studies in Enthnomethodology, N.J.Prentice-Hall. 1967.

9. Que l'on pourrait tenter de traduire par « réflexivité »?

10. JL. Le Moigne. "Voir la nature avec les yeux de l'art » in Systèmes naturels, systèmes artificiels, ouvrage collectif dirigé par Franck Tinland. Champ Vallon. 1991 p. 129.

11. Cité par JL. Le Moigne, cf note (10)

12. Louis Outre, «D'un modèle épistémologique de la communication à un modèle praxéologique » p. 88-89 in revue Réseaux, n 46-47 CNET 1991.

13. Patrice Flichy, L'innovation technique. Éditions La Découverte. 1995

14. Louis Quéré, "D’un modèle épistémologique de la communication à un modèle praxéologique » p. 88-89 in revue Réseaux, n46-47.CNET. 1991.

15. Alain Renault. « Le sujet de la communication », revue Réseaux, n 46-47, CNET, 1991

16. Cl. Allegre. La défaite de Platon, Seuil. 1995. p 408

17. «Le positif, c'est le réel » A. Comte. " Discours sur l'esprit positif», in Traité philosophique d'astronome populaire, 1844. cite par Le Moigne dans son article «Voir la nature avec les yeux de l'art » déjà cité cf infra note (10)

18. Winograd. "representation and understanding ", studiti in cognitivi science, academic press, New York, 1975, cité par Michel de Fomel «Qu'est-ce qu'un expert » in revue Réseaux, CNET. n - 43.

19. Braudel, Annales ESC. Vol. 13 n 4. Oct-déc 1998

20. Philippe Breton, article "Théorie de l'information ", in Dictionnaire de la Communication, p. 214 du tome 2, dirigé par L. Sfez

21. J. CL. Abric. Psychologie de la communication. Armand Colin. 1996

22. A. Pelissier et A. Tête, Sciences cognitives : les textes fondateurs de 1943 à 1950, PUF, 1995

23. Eliséo Véron "Pour en finir avec la communication ", in revue Réseaux. n 46/47, CNET. 1991

24. Sergio Vasquez Bronfman «Approche langage/action et efficacité des réseaux de conversations récurrentes ", in revue Système d'information et management, avril 1996

25. "Je sais bien que l'esprit est toujours fécond en mode de comprendre. Je le dis souvent aux personnes que je contrôle - faites surtout bien attention à ne pas comprendre le malade, il n'y a rien qui vous perde comme ça » J. Lacan. Livre II du Séminaire, Seuil, p. 109.

26. Lacan idem p. 26

27. Eliséo Véron, Revue Réseaux n 46-47 déjà cité note (23)

\section{AUTEUR}

\section{FRANÇOISE COLIN}

Université de la Méditerranée IUT Aix Provence 